Supporting Information

\title{
Multiple Self-Trapped Emissions in the Lead-free Halide $\mathrm{Cs}_{3} \mathrm{Cu}_{2} \mathrm{I}_{5}$
}

\author{
Haijie Chen, ${ }^{1}$ Joao M. Pina, ${ }^{1}$ Fanglong Yuan, ${ }^{2}$ Andrew Johnston, ${ }^{1}$ Dongxin Ma,,${ }^{1}$ Bin \\ Chen, ${ }^{1}$ Ziliang Li, ${ }^{1}$ Antoine Dumont,,${ }^{2}$ Xiyan Li, ${ }^{1}$ Yanan Liu, ${ }^{3}$ Sjoerd Hoogland, ${ }^{1}$ Zoltán \\ Zajacz, ${ }^{3}$ Zhenghong Lu, ${ }^{2}$ and Edward H. Sargent ${ }^{*, 1}$
}

${ }^{I}$ The Edward S. Rogers Department of Electrical and Computer Engineering, University of Toronto, Toronto, Ontario, Canada M5S 3G4

${ }^{2}$ Department of Materials Science and Engineering, University of Toronto, Toronto, Ontario, Canada M5S 3E4

${ }^{3}$ Department of Earth Sciences, University of Toronto, Toronto, Ontario, Canada M5S 3B1 


\section{Experimental Section}

Precursor Materials. Cesium iodide (CsI, 99.999\%), copper(I) iodide (CuI, 99.999\%), dimethyl sulfoxide (DMSO, 99.9\%), dimethylformamide (DMF, 99.8\%), poly(2hydroxyethyl methacrylate) (poly-HEMA), methanol (MeOH, 99.9\%), and Nafion perfluorinated resin solution (PFI, 5 wt.\% in mixture of lower aliphatic alcohols and water, contains $45 \%$ water) were purchased from Sigma-Aldrich and used without further modification.

Bulk Sample Synthesis. Single crystals of $\mathrm{CsCu}_{2} \mathrm{I}_{3}$ were grown through the slow vapor saturation of an antisolvent (VSA). ${ }^{1}$ A clear $1 \mathrm{M}$ stoichiometric $\mathrm{CsCu}_{2} \mathrm{I}_{3}$ solution was prepared by dissolving CsI and CuI in a mixture of DMF and DMSO $(3: 1 \mathrm{v} / \mathrm{v})$ in a $\mathrm{N}_{2^{-}}$ filled glovebox under stirring for $2 \mathrm{~h}$. $\mathrm{MeOH}$ was then slowly titrated until white powders formed in the solution. The saturated solution was further filtered with PTFE $0.22 \mu \mathrm{m}$ pore size syringe filters. The filtered solution, covered by a paraffin film with a small hole in the center, was transferred into a vessel filled with $\mathrm{MeOH}$. After keeping the sealed vessel in an oven at $60^{\circ} \mathrm{C}$ for 2 days, needle-like $\mathrm{CsCu}_{2} \mathrm{I}_{3}$ single crystals with length $\sim 1 \mathrm{~cm}$ were obtained in the bottom.

$\mathrm{Cs}_{3} \mathrm{Cu}_{2} \mathrm{I}_{5}$ polycrystals were synthesized by the stoichiometric reaction of $\mathrm{CsI}$ and CuI. $^{2}$ A mixture of $272.8 \mathrm{mg} \mathrm{CsI}$ and $266.6 \mathrm{mg} \mathrm{CuI}$ was sealed in a silica tube $(10 \mathrm{~mm}$ diameter) under vacuum $\left(10^{-3} \mathrm{mbar}\right)$ and subsequently transferred into a furnace. The furnace was heated to $400^{\circ} \mathrm{C}$ for $4 \mathrm{~h}$ and dwelled for $5 \mathrm{~h}$. The final $\mathrm{Cs}_{3} \mathrm{Cu}_{2} \mathrm{I}_{5}$ ingot was obtained without any impurity after cooling the tube to room temperature for $20 \mathrm{~h}$. 
Powder X-ray Diffraction (PXRD). Data was collected on a Rigaku Miniflex powder X-ray diffractometer with $\mathrm{Cu} \mathrm{K} \alpha$ radiation $(\lambda=1.5406 \AA)$ with a voltage of $40 \mathrm{kV}$ and a current of $15 \mathrm{~mA}$.

Photoluminescence (PL) and Absorption Measurements. PL spectra were measured using a Horiba Fluorolog time-correlated single photon counting system equipped with UV/vis/NIR photomultiplier tube detectors, dual grating spectrometers, and a monochromatized xenon lamp excitation source. A source with $\lambda=300 \mathrm{~nm}$ was adopted to obtain the transient PL signal. The other settings are as following: bandpass values of 10 and $5 \mathrm{~nm}$ for the excitation and emission slits, respectively, step increments of $1 \mathrm{~nm}$ and integration times of $0.5 \mathrm{~s}$ per data point. The PLQY values were evaluated by coupling a Quantum-Phi integrating sphere to the Fluorolog system through optical fibres. Our experimental setting ensured that the spectra had high signal-to-noise ratios and provided excitation intensities in the range of $1-30 \mathrm{~mW} \mathrm{~cm}{ }^{-2}$. Optical absorption spectra were obtained in a Lambda $950 \mathrm{UV}$-vis-IR spectrophotometer.

Temperature- and Power-dependent PL Spectra. Samples were optically excited using a $315 \mathrm{~nm}$ fs laser source with a repetition rate of $5000 \mathrm{~Hz}$. The laser source consisted of a Yb:KGW regenerative amplifier (Pharos, Light Conversion) with 1030nm fundamental which is passed through an optical parametric amplifier (Orpheus, Light Conversion) to generate the $315 \mathrm{~nm}$ pump light pulse. Excitation power was controlled using a continuously variable neutral density filter wheel. The spectral data is collected using a UVvis USB 2000+ spectrometer (Ocean Optics). For temperature-dependent measurements, a VPF liquid nitrogen cooled cryostat (Janis Research) was used. 
Inelastic Neutron Scattering (INS) measurements were done using the time-of-flight direct geometry spectrometer SEQUOIA at the Spallation Neutron Source (SNS), Oak Ridge National Laboratory. ${ }^{3}$ INS spectra were collected at several incident energies $\left(E_{\mathrm{i}}=\right.$ $18,50,160$ and $600 \mathrm{meV}$ ) to provide high energy resolution, $\sim 1-5 \%$ of the $E_{\mathrm{i}}$, in wide range of energy transfer. The measurements were done at temperature $T=5 \mathrm{~K}$ using a bottom-loading closed-cycle helium refrigerator. Background spectra for an empty container were collected and subtracted from the sample data. The INS data were corrected for detector efficiency and transformed from time-of-flight and instrument coordinates to the dynamic structure factor $\mathrm{S}(\mathrm{Q}, \mathrm{E})$, where $\mathrm{Q}$ and $\mathrm{E}$ are neutron momentum and energy transfer. In one-phonon approximation, the measured neutron energy-loss $S(Q, E)$ spectrum relates to the generalized phonon density of states $G(E)$ as $\mathrm{S}(\mathrm{Q}, \mathrm{E}) \sim \mathrm{Q}^{2} \mathrm{G}((\mathrm{E})[\mathrm{n}(\mathrm{E}, \mathrm{T})+1] / \mathrm{E}$, where $\mathrm{n}(\mathrm{E}, \mathrm{T})$ is the population Bose factor.

Atomic Force Microscopy (AFM) Measurements. The surface morphologies of the $\mathrm{Cs}_{3} \mathrm{Cu}_{2} \mathrm{I}_{5}$ films were measured using ScanAsyst in air. The contilever used is Scanasystair, a silicon tip on a nitride lever with spring constant of $\sim 0.4 \mathrm{~N} \mathrm{~m}^{-1}$. Samples were prepared in the glovebox under the same spin-coating conditions as the LED devices.

LED Fabrication. Glass substrates with pre-patterned indium tin oxide (ITO), with a conductivity of $100 \Omega \mathrm{sq}^{-1}$, were cleaned by Triton, deionized water, acetone, and isopropanol in ultrasonic bath. The substrates were then subjected to $\mathrm{O}_{2}$ plasma for $5 \mathrm{~min}$. PEDOT:PSS was mixed with PFI $(1: 1 \mathrm{v} / \mathrm{v})$ and stirred for $2 \mathrm{~h}$ to form a homogenous solvent. It was filtered through a $0.45 \mu \mathrm{m}$ PVDF (polyvinylidene difluoride) filter and spin-coated on the substrates, followed by 20 min annealing at $150^{\circ} \mathrm{C} .0 .5 \mathrm{M} \mathrm{Cs}_{3} \mathrm{Cu}_{2} \mathrm{I}_{5}$ solution was mixed with $10 \mathrm{mg} / \mathrm{mL}$ poly-HEMA to prepare the precursor. The precursor 
solution $(200 \mu \mathrm{L})$ was spin-coated at $5000 \mathrm{rpm}$ for $90 \mathrm{~s}$, followed by annealing on a hot plate at $100^{\circ} \mathrm{C}$ for $1 \mathrm{~h}$ in a $\mathrm{N}_{2}$-filled glovebox. The substrates were then transferred to a thermal evaporator where the desired thickness of 50nm TPBi, $1 \mathrm{~nm}$ lithium fluoride and $120 \mathrm{~nm}$ aluminum layers were consecutively deposited through a shadow mask, at a vacuum pressure of less than $10^{-4} \mathrm{~Pa}$. The device active area was $6.14 \mathrm{~mm}^{2}$ as defined by the overlapping area of the ITO and Al electrodes.

LED characterization. Devices were tested under ambient conditions. Luminancevoltage and the current density-voltage characteristics were monitored via a computercontrolled Keithley 2400 Source Meter. EQEs were determined as the number of emitted photons to the number of injected electrons. The measurement was calibrated using efficient organic LED devices. 
a

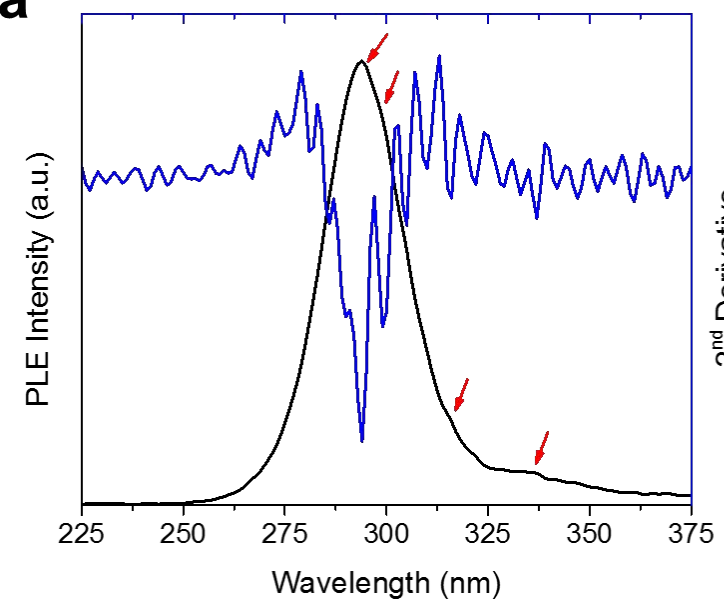

b

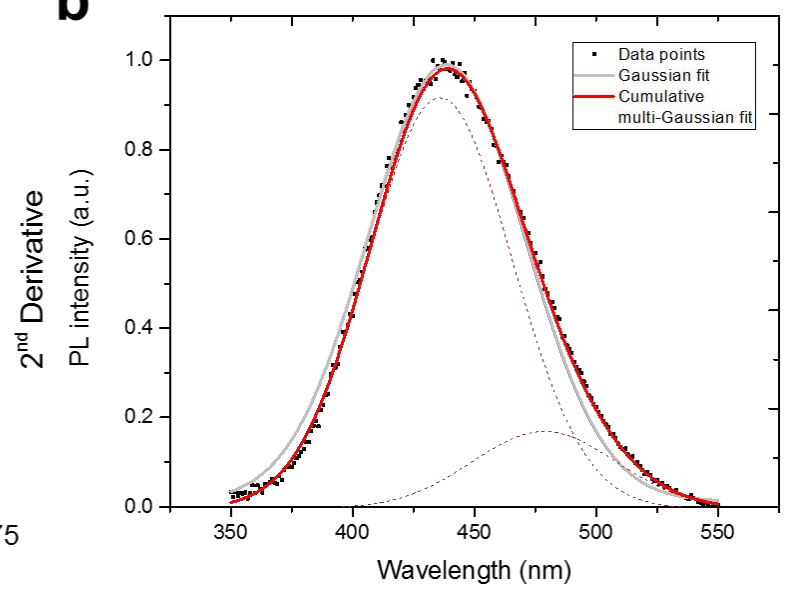

Figure S1. (a) The second derivative of the photoluminescence excitation (PLE). (b) The fittings of the PL spectra by an exponentially modified Gaussian by multipule Gaussian distributions. 


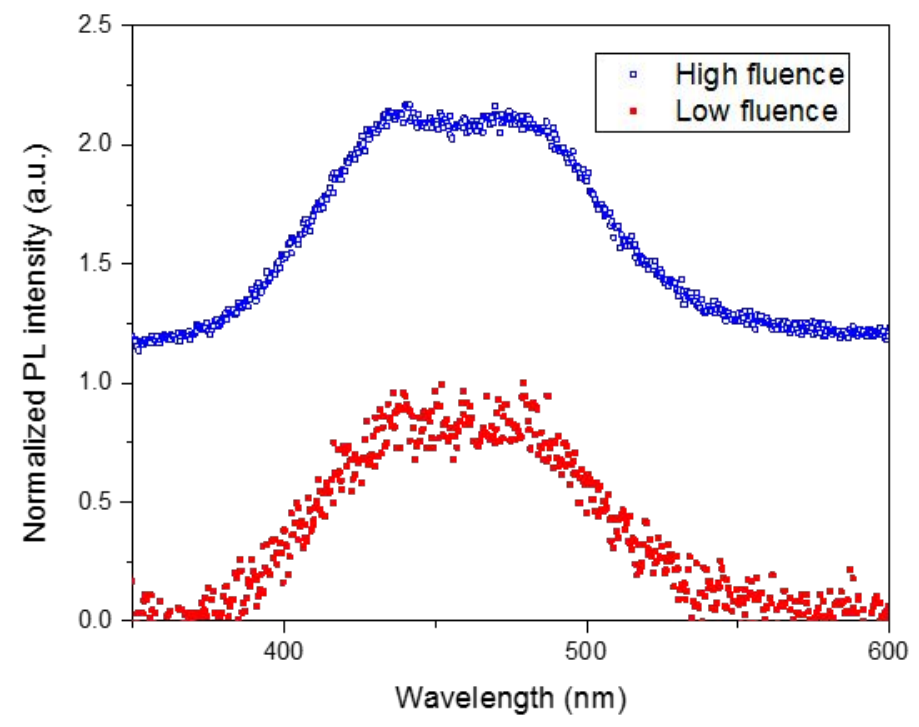

Figure S2. Normalized multiple emission peaks at high and low excitation fluences. 

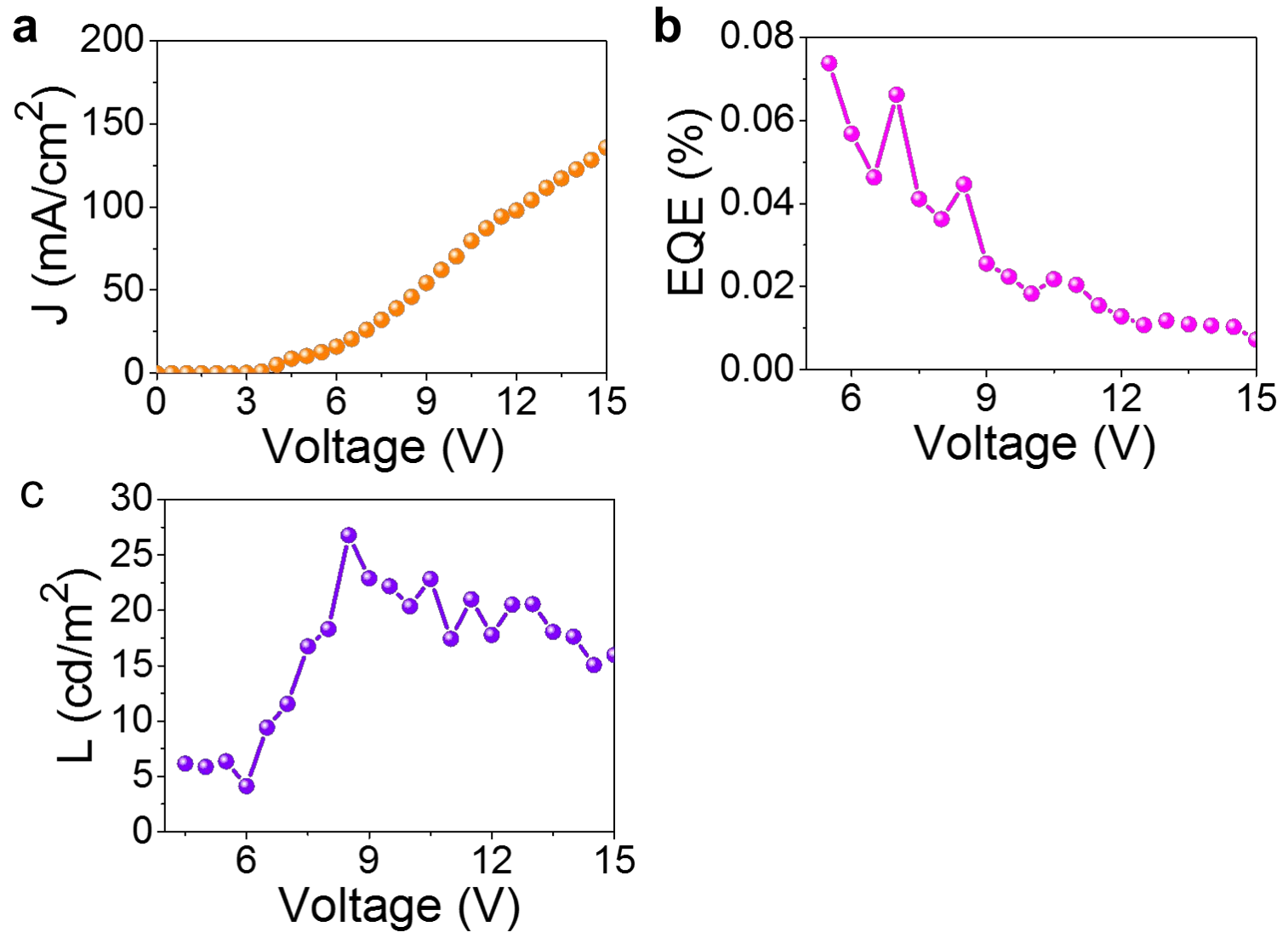

Figure S3. (a) Current density, (b) EQE, and (c) luminance versus applied voltage characteristics of the device with $\mathrm{Cs}_{3} \mathrm{Cu}_{2} \mathrm{I}_{5}$ emission layer. 


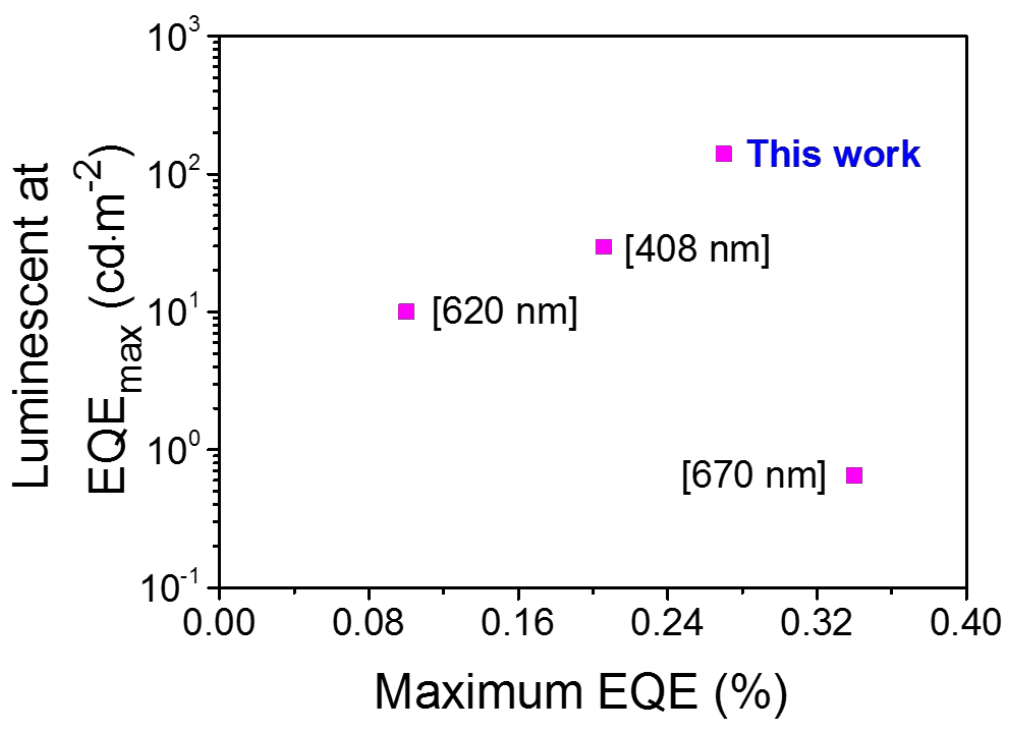

Figure S4. Comparative performance table of lead-free halide LEDs. 


\section{References}

1. Rakita, Y.; Kedem, N.; Gupta, S.; Sadhanala, A.; Kalchenko, V.; Böhm, M. L.; Kulbak, M.; Friend, R. H.; Cahen, D.; Hodes, G., Low-temperature solution-grown $\mathrm{CsPbBr}_{3}$ single crystals and their characterization. Crystal Growth \& Design 2016, 16 (10), 5717-5725.

2. Roccanova, R.; Yangui, A.; Nhalil, H.; Shi, H.; Du, M.-H.; Saparov, B., NearUnity Photoluminescence Quantum Yield in Blue-Emitting $\mathrm{Cs}_{3} \mathrm{Cu}_{2} \mathrm{Br}_{5-\mathrm{x}} \mathrm{I}_{\mathrm{x}}(0 \leq \mathrm{x} \leq 5)$. ACS Applied Electronic Materials 2019, 1 (3), 269-274.

3. Granroth, G. E.; Kolesnikov, A. I.; Sherline, T. E.; Clancy, J. P.; Ross, K. A.; Ruff, J. P. C.; Gaulin, B. D.; Nagler, S. E. In SEQUOIA: A newly operating chopper spectrometer at the SNS, Journal of Physics: Conference Series, IOP Publishing: 2010; $\mathrm{p}$ 012058. 